\title{
Prevalência de transtornos psiquiátricos em pacientes em hemodiálise no estado da Bahia
}

\author{
Prevalence of psychiatric disorders in patients in hemodialysis \\ in the state of Bahia
}

Jose A. Moura Junior, Carlos A. Marcilio de Souza, Irismar Reis de Oliveira, Roberta Oliveira Miranda

\begin{abstract}
Resumo
Introdução: Limitações da qualidade de vida dos pacientes com insuficiência renal crônica (IRC) em programa de hemodiálise estão associadas a maior prevalência de transtornos psiquiátricos. O diagnóstico precoce e preciso pode contribuir na elaboração de estratégias de tratamento. Objetivo: Estudar a prevalência e o padrão dos transtornos psiquiátricos em pacientes hemodialisados, identificando variáveis relacionadas com sua ocorrência. Métodos: Foram estudados 244 pacientes e analisadas as variáveis associadas à ocorrência de transtornos psiquiátricos. Os pacientes foram avaliados através do Mini-International Neuropsychiatric Interview (MINI). Resultados: Pelo menos um diagnóstico psiquiátrico foi apresentado por 91 pacientes $(37,3 \%)$. Os de ocorrência mais freqüente foram distimia $(17,6 \%)$, risco de suicídio (16,4\%) e episódio depressivo maior (8,6\%). O sexo feminino apresentou maior risco de transtornos psiquiátricos (razão de chance $[R C]=2,77$; intervalo de confiança [IC] 95\%: 1,42-5,41). Os pacientes viúvos, separados e solteiros apresentaram maior risco de transtornos psiquiátricos do que os casados ( $R C=5,507 ;$ IC 95\%: 1,348-22,551). Aqueles com menos de dois anos em diálise apresentaram risco maior ( $\mathrm{RC}=2,075$; IC 95\%: 1,026-4,197). Os pacientes com clearance fracional de uréia (KtN) abaixo de 0,9 tiveram maior chance de apresentar transtornos psiquiátricos ( $R C=3,955$; IC 95\%: 1,069-11,012). Conclusões: A prevalência de transtornos psiquiátricos foi alta. Os transtornos afetivos foram os mais freqüentes. Nas mulheres e naqueles com KtV baixo, o risco foi maior. Nos pacientes casados e naqueles com mais de dois anos em tratamento o risco foi menor.

Palavras-chave: transtornos psiquiátricos, hemodiálise; Mini-International Neuropsychiatric Interview.
\end{abstract}

\begin{abstract}
Introduction: Limiting factors of dialysis patients' quality of life are associated with high rates of psychiatric disease. Since effective treatment of psychiatric diseases can positively affect dialysis patients' prognosis and survival, there is a need for an early diagnosis. In Brazil only few studies have measured the prevalence of these disorders. Objective: Study the prevalence and pattern of psychiatric disorders in chronic hemodialysis patients and assess the association between these diseases and some variables. Methods: Two hundred forty-four patients were enrolled in two nephrology units at the state of Bahia, Brazil. The Mini-International Neuropsychiatric Interview (MINI) was used in all patients. Results: Ninetyone patients (37.3\%) presented at least one psychiatric diagnosis. The most common disorders included dysthymia (17.6\%), risk of suicide (16.4\%), and major depressive episode (8.6\%). Women had higher risk of mental disorders than men ( $R C=2.77$; confidence interval [CI] 95\%: 1.42-5.41). Widowed, divorced and single patients had higher risk than married patients $(\mathrm{RC}=5.507 ; \mathrm{Cl} 95 \%$ : 1.348-22.551). Patients on treatment for less than two years had higher risk $(\mathrm{RC}=2.075$; $\mathrm{Cl}$ 95\%: 1.026-4.197). Those with $\mathrm{Kt} / \mathrm{V}$ below 0.9 were at higher risk than those with a higher $\mathrm{Kt} / \mathrm{V}(\mathrm{RC}=3.955 ; \mathrm{Cl} 95 \%$ : 1.069-11.012). Conclusions: The prevalence of psychiatric disorders in patients on dialysis was high (37.3\%). Affective disorders were the most common ones. Women and patients with $\mathrm{Kt} / \mathrm{V}$ below 0.9 were at increased risk. The risk was lower in patients at dialysis program for longer than two years and in married patients.

Key words: psychiatric diseases, hemodialysis, Mini-International Neuropsychiatric Interview.
\end{abstract}

Fundação para o Desenvolvimento das Ciências (Moura Junior JA, Souza CAM)

Universidade Federal da Bahia (UFBA) (Oliveira IR, Miranda RO)

Hospital Universitário Professor Edgard Santos (HUPES) (Oliveira IR)

Recebido Trabalho baseado na dissertação para obtenção de Grau de Mestre com o título de Prevalência de Transtornos Psiquiátricos em Pacientes em Hemodiálise no Estado da Bahia (em 2002), apresentada na 23-08-06 Fundação para Desenvolvimento das Ciências. 


\section{Introdução}

Insuficiência renal crônica (IRC), expressão que se aplica a toda condição patológica de instalação gradual com potencial para deteriorar (em graus variáveis) a capacidade funcional dos rins, é uma condição de impacto psicossocial que constitui problema de saúde pública de grande magnitude, vez que o número estimado de pacientes em programas de diálise em todo o mundo ultrapassa um milhão, com a prevalência da patologia se mantendo na média de 350 pacientes por milhão de habitantes (Dialysis Statistics; U.S. Renal Data System, 2000). Assim sendo, os portadores de IRC ficam dependentes de uma máquina para sobreviver, mas esse quadro se apresenta numa extensão sem precedente na história da tecnologia médica.

Até o início dos anos 1960 o desfecho fatal era a regra na totalidade dos casos de IRC devido à inexistência de métodos de diálise (U.S. Renal Data System, 2000). Entretanto, a história natural dessa enfermidade modificou-se radicalmente com a introdução da hemodiálise e com o desenvolvimento do transplante, adventos que contribuíram para melhorar substancialmente o prognóstico (Lloyd, 1991). A preocupação com os portadores de IRC era quase que exclusivamente 0 prolongamento da sobrevida, mas hoje ela também objetiva melhorar a qualidade de vida dos pacientes. Nessa conjuntura, conforme a ênfase na qualidade de vida, percebe-se facilmente a importância de se identificar problemas psiquiátricos, que estão diretamente relacionados a um melhor prognóstico (Finkelstein e Finkelstein, 2000).

Não há estudos no Brasil que mensurem a real prevalência desses transtornos no âmbito das unidades de diálise, assim como a sua associação com variáveis sociodemográficas e clínicas. Este estudo pretendeu identificar possíveis casos de transtornos psiquiátricos no âmbito de duas unidades de diálise, utilizando o Mini-International Neuropsychiatric Interview (MINI), um questionário muito utilizado pelos psiquiatras capaz de reconhecer substancial parcela de indivíduos com alterações de saúde mental, sendo compatível com critérios diagnósticos internacionais atuais, e que prioriza os mais prevalentes transtornos psiquiátricos (Sheehan et al., 1998).

O objetivo dessa pesquisa foi estudar a prevalência e o padrão dos transtornos psiquiátricos em pacientes portadores de IRC em programa de hemodiálise, correlacionando-os com variáveis clínicas e sociodemográficas, bem como verificando a possível associação dessas variáveis com a ocorrência dos transtornos psiquiátricos encontrados.

\section{Métodos}

Estudo de coorte transversal foi realizado em duas clínicas de nefrologia no estado da Bahia: uma em Salvador e outra em Feira de Santana, distante 108 km da capital. A amostra foi constituída por 244 pacientes que faziam hemodiálise regular nesses serviços, e foram escolhidos de acordo com tabela de randomização. Os convidados concordaram em participar do estudo e assinaram o termo de consentimento livre e esclarecido. O protocolo do estudo, realizado entre outubro de 2001 e fevereiro de 2002, foi aprovado pelo Comitê de Ética em Pesquisa do Centro de Pesquisas Gonçalo Moniz (CPqGM) da Fundação Oswaldo Cruz (FIOCRUZ).

Todos os pacientes dialisavam três dias por semana, em sessões de quatro horas cada, e os critérios de inclusão foram: ser portador de IRC terminal; estar em programa de hemodiálise nas unidades do estudo há mais de 30 dias; ter concordado em participar do estudo assinando o termo de consentimento livre e esclarecido.

As entrevistas foram realizadas por uma psiquiatra treinada na utilização do MINI e aconteceram nas próprias clínicas, em consultórios individuais, antes ou depois da hemodiálise dos pacientes. As variáveis sociodemográficas foram sexo, idade, estado civil, ocupação e moradia. As variáveis clínicas foram tempo em diálise, hemoglobina $(\mathrm{Hb})$ e clearance fracional de uréia (Kt/V), que foi utilizado como parâmetro para se definir a dose de diálise a ser prescrita (Hakim et al., 1994).

Para a construção do banco de dados visando as análises estatísticas, foram utilizados os programas Epilnfo (versão 6.4) e o SPSS Windows (versão 9.0). Foram observadas as prevalências dos transtornos, segundo as co-variáveis de interesse, e analisadas as diferenças entre as categorias através do qui-quadrado $\left(\chi^{2}\right)$, sendo estimadas as magnitudes dessas associações através das medidas de associação (razão de chance $[R C]$ ) e os seus respectivos intervalos de confiança (IC) a 95\%. O nível de significância adotado nos testes estatísticos foi de $5 \%(p<0,05)$.

\section{Resultados}

O MINI foi aplicado em 244 pacientes, o que representou $57,5 \%$ da população das duas clínicas de diálise, com tempo médio de aplicação de $12,11 \pm 1,99$ minuto. $A$ Tabela 1 sumariza as características sociodemográficas e clínicas da amostra, com os percentuais encontrados e seus respectivos ICs.

Os diagnósticos psiquiátricos mais comuns encontrados na amostra foram:

- distimia em 43 pacientes (17,6\%);

- risco de suicídio em 40 pacientes (16,4\%);

- episódio depressivo maior em 21 pacientes (8,6\%);

- fobia social em 13 pacientes (5,3\%).

A Tabela 2 resume esses números. Já a prevalência dos que apresentaram apenas um diagnóstico foi 22,1\% (54 pacientes); 27 (11,1\%) apresentaram dois diagnósticos; seis, três diagnósticos; dois tiveram quatro transtornos e outros dois pacientes apresentaram cinco transtornos. Quando considerados em conjunto, mais do que um terço, ou seja, 91 pacientes $(37,3 \%)$ apresentaram algum transtorno psiquiátrico. A Figura 1 apresenta essas proporções.

Entre as variáveis sociodemográficas avaliadas, gênero e estado civil estão associados aos transtornos psiquiátricos diagnosticados, com resultados estatisticamente significantes em torno de 5\%. Das 101 mulheres entrevistadas, 47,5\% apresentaram pelo menos um diagnóstico, enquanto entre os 143 homens esse percentual foi de $30,1 \%(p=0,0055)$, portanto uma diferença estatisticamente significante. Dos 121 
Tabela 1. Variáveis sociodemográficas e clínicas dos pacientes em programas de hemodiálise

\begin{tabular}{|c|c|c|c|c|c|c|c|}
\hline \multicolumn{4}{|c|}{ Variáveis sociodemográficas } & \multicolumn{4}{|c|}{ Variáveis clínicas } \\
\hline Sexo & $n$ & $\%$ & IC $95 \%$ & Tempo & $n$ & $\%$ & IC $95 \%$ \\
\hline Masculino & 143 & 58,6 & $52,2-64,8$ & $\leq 12 m$ & 44 & 18 & $13,4-23,4$ \\
\hline \multirow[t]{2}{*}{ Feminino } & 101 & 41,4 & $35,2-47,9$ & 13 a $24 m$ & 49 & 20,1 & $15,2-25,7$ \\
\hline & & & & 25 a $36 m$ & 29 & 11,9 & $8,1-16,6$ \\
\hline \multicolumn{8}{|c|}{ Faixa etária (anos) } \\
\hline$\leq 20$ & 12 & 4,9 & $2,6-8,4$ & 37 a 48m & 31 & 12,7 & $8,8-17,5$ \\
\hline 21 a 40 & 97 & 39,8 & $33,6-46,2$ & 49 a $60 m$ & 34 & 13,9 & $9,8-18,8$ \\
\hline 41 a 60 & 89 & 36,5 & $30,4-42,9$ & $\geq 61 \mathrm{~m}$ & 57 & 23,4 & $18,2-29,2$ \\
\hline$\geq 61$ & 44 & 18 & $13,4-23,4$ & & & & \\
\hline \multicolumn{4}{|l|}{ Estado civil } & $\mathrm{Hb}(\mathrm{g} / \%)$ & & & \\
\hline Casado & 121 & 49,6 & $43,2-56$ & $\leq 9$ & 65 & 26,6 & $21,2-32,7$ \\
\hline Solteiro & 102 & 41,8 & $35,5-48,3$ & 9,1 a 13 & 161 & 66 & $59,7-71,9$ \\
\hline Viúvo & 10 & 4,1 & $2-7,4$ & $\geq 13,1$ & 16 & 6,6 & $3,8-10,4$ \\
\hline Separado & 11 & 4,5 & $2,3-7,9$ & & & & \\
\hline \multicolumn{4}{|l|}{ Ocupação } & $\mathrm{Kt} / \mathrm{V}$ & & & \\
\hline Aposentado & 141 & 57,8 & $51,3-64,1$ & $\leq 0,9$ & 17 & 7 & $4,1-10,9$ \\
\hline Estudante & 24 & 9,8 & $6,4-14,3$ & 0,91 a 1,2 & 70 & 28,7 & $23,1-34,8$ \\
\hline Doméstico & 55 & 22,5 & $17,3-28,3$ & $\geq 1,21$ & 152 & 62,3 & $58,9-68,4$ \\
\hline Empregado & 19 & 7,8 & $4,8-11,9$ & Total & 244 & 100 & \\
\hline \multicolumn{8}{|l|}{ Domicílio } \\
\hline Urbano & 185 & 76,1 & $69,9-81,1$ & & & & \\
\hline Rural & 58 & 23,9 & $18,6-29,6$ & & & & \\
\hline Total & 244 & 100 & & & & & \\
\hline
\end{tabular}

m: meses; Hb: hemoglobina; Kt/V: clearance fracional de uréia.

Tabela 2. Prevalência dos transtornos psiquiátricos encontrados na amostra

\begin{tabular}{llllllll}
\hline Transtornos & $n$ & Presente (\%) & IC 95\% & $n$ & Ausente (\%) & IC 95\% & Total \\
\hline Episódio depressivo maior & 21 & 8,6 & $5,1-12,1$ & 223 & 91,4 & $87,9-94,9$ & 244 \\
Episódio depressivo passado & 5 & 2 & $0,3-3,8$ & 235 & 96,3 & $96,1-99,7$ & 244 \\
Episódio depressivo com características & 8 & 3,3 & $1-5,5$ & 236 & 96,7 & $94,5-99$ & 244 \\
melancólicas & & & & & & & \\
Distimia & 43 & 17,2 & $12,8-22,4$ & 201 & 82,4 & $77,6-87,2$ & 244 \\
Risco de suicídio & 40 & 16,4 & $11,7-21$ & 204 & 83,6 & $79-88,3$ & 244 \\
Episódio hipomaníaco & - & - & - & 244 & 100 & - & 244 \\
Transtorno de pânico & - & - & - & 244 & 100 & - & 244 \\
Agorafobia & 3 & 1,2 & $0,2-2,6$ & 241 & 98,8 & $97,4-100$ & 244 \\
Fobia social & 13 & 5,3 & $2,5-8,1$ & 231 & 94,7 & $91,9-97,5$ & 244 \\
Transtorno obsessivo-compulsivo & - & - & - & 244 & 100 & - & 244 \\
Transtorno de estresse pós-traumático & - & - & - & 244 & 100 & - & 244 \\
Dependência/abuso de álcool & 3 & 1,2 & $0,2-2,6$ & 241 & 98,8 & $97,4-100$ & 244 \\
Dependência/abuso de substâncias & - & - & - & 244 & 100 & - & 244 \\
Síndrome psicótica & 3 & 1,2 & $0,2-2,6$ & 241 & 98,8 & $97,4-100$ & 244 \\
Anorexia nervosa & - & - & - & 244 & 100 & - & 244 \\
Bulimia nervosa & 1 & 0,4 & $0,4-1,2$ & 243 & 99,6 & $98,8-100$ \\
Transtorno de ansiedade generalizada & 5 & 2 & $0,3-3,8$ & 239 & 97,9 & $96,2-99,7$ & 244 \\
Transtorno da personalidade anti-social & 1 & 0,4 & $0,4-1,2$ & 243 & 98,8 & $98,8-100$ & 244 \\
\hline
\end{tabular}


Figura 1. Prevalência dos transtornos psiquiátricos encontrados quando considerados em conjunto

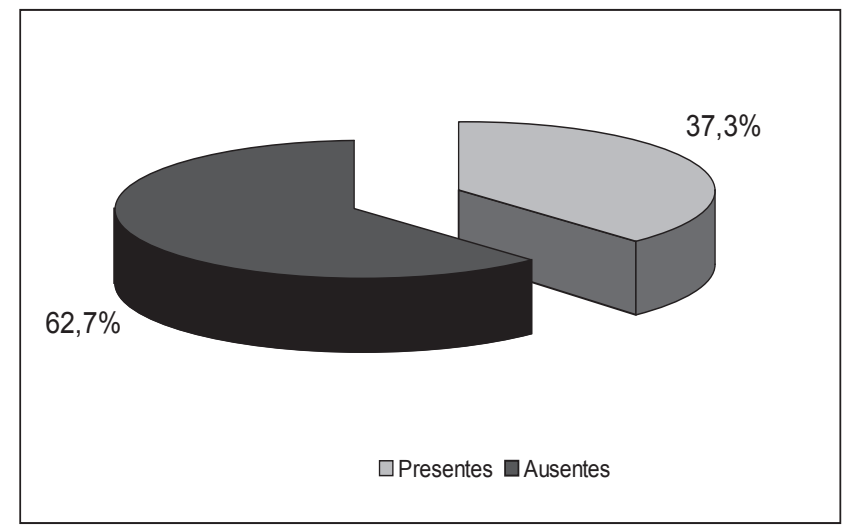

pacientes casados examinados, 29,8\% foram diagnosticados como portadores de pelo menos um transtorno. Entre os não-casados (102 solteiros, 11 separados e 10 viúvos), observou-se prevalência de 55 pacientes com pelo menos um transtorno $(p=0,023)$ estatisticamente significante.

Das três variáveis clínicas selecionadas para a avaliação da associação com transtornos psiquiátricos, apenas o Kt/V foi significantemente associado. A prevalência observada nos 17 pacientes com Kt/V abaixo de 0,9 foi $64 \%$, significativamente maior que a ocorrida nos 222 pacientes com a medida acima de 0,91 , que foi $32,7 \%(p=0,035)$. A magnitude da associação entre as variáveis e os transtornos psiquiátricos, com suas RCs não ajustadas e ajustadas pelas demais co-variáveis presentes, está demonstrada na Tabela 3.

O sexo feminino apresentou maior chance de apresentar transtornos psiquiátricos do que o masculino $(R C=2,11$; IC $\left.95 \%: 1,24-3,58^{*}\right)$. Após considerar as diversas co-variáveis, 0 risco aumentou para cerca de três vezes $(R C=2,77$; IC 95\%: 1,42-5,41). Os pacientes solteiros apresentaram uma vez e meia de mais chance de diagnóstico de transtornos psiquiátricos, quando em comparação com os casados ( $R C=1,65$; IC 95\%: 0,949-2,877). Por sua vez os viúvos e os separados tinham, respectivamente, cinco a três vezes mais chances de transtornos, quando em comparação com os casados $(R C=5,507$; IC 95\%: 1,348-22,510*) e $(R C=2,831$; IC 95\%: $0,812-9,881)$.

Das variáveis clínicas analisadas, tanto o Kt/V como o tempo em diálise foram significantes e independentemente associados. Os pacientes com menos de dois anos em diálise apresentaram duas vezes mais chances de ocorrência de transtornos psiquiátricos na análise bivariada $(R C=2,075$; IC 95\%: 1,026-4,197). Na análise multivariada, com controle das outras variáveis, o risco foi quase três vezes maior $(R C=2,673$; IC 95\%: 1,169-6,111).

Também em relação ao índice de adequação à diálise (Kt/V), a medida de risco aparece quando são considerados os pacientes com maior chance de ocorrência de transtornos, ou seja, aqueles com Kt/V abaixo de 0,9. Esses pacientes tiveram a razão de risco estimada em quatro vezes $(R C=3,955$; IC 95\%: 1,069-11,012).
Tabela 3. Razões de chance após avaliações bivariada e multivariada das variáveis sociodemográficas e clínicas

\begin{tabular}{|c|c|c|}
\hline \multicolumn{3}{|c|}{ Variáveis sociodemográficas } \\
\hline & $\begin{array}{l}\text { Bivariada RC } \\
\text { (IC 95\%) }\end{array}$ & $\begin{array}{l}\text { Multivariada RC } \\
\text { (IC 95\%) }\end{array}$ \\
\hline \multicolumn{3}{|l|}{ Sexo } \\
\hline Masculino & $2,11(1,24-3,58)^{*}$ & $2,77(1,42-5,41)^{\star}$ \\
\hline Feminino & 1 & 1 \\
\hline \multicolumn{3}{|l|}{ Faixa etária } \\
\hline$\leq 20$ anos & $0,281(0,058-1,359)$ & $0,124(0,02-0,773)$ \\
\hline 21 a 40 anos & $0,905(0,5-1,63)$ & $0,941(0,473-1,873)$ \\
\hline 41 a 60 anos & 1 & 1 \\
\hline$\geq 61$ anos & $0,589(0,272-1,276)$ & $0,611(0,247-1,512)$ \\
\hline \multicolumn{3}{|l|}{ Estado civil } \\
\hline Casado & 1 & 1 \\
\hline Solteiro & $1,653(0,949-2,877)^{\star}$ & $2,076(1,057-4,075)^{*}$ \\
\hline Viúvo & $5,509(1,348-22,51)^{\star}$ & $6,12(1,203-31,122)$ \\
\hline Separado & $2,833(0,812-4,881)$ & $2,126(0,472-9,575)$ \\
\hline \multicolumn{3}{|l|}{ Ocupação } \\
\hline Empregado/estudante & 1 & 1 \\
\hline Aposentado & $1,084(0,54-2,16)$ & $0,709(0,3-1,677)$ \\
\hline \multicolumn{3}{|l|}{ Moradia } \\
\hline Urbana & 1 & 1 \\
\hline Rural & $0,8(0,44-1,47)$ & $0,889(0,441-1,793)$ \\
\hline \multicolumn{3}{|c|}{ Variáveis clínicas } \\
\hline \multicolumn{3}{|l|}{ Tempo em diálise } \\
\hline$\leq 24 \mathrm{~m}$ & $2,075(1,026-4,197)^{\star}$ & $2,673(1,169-6,111)^{\star}$ \\
\hline 25 a $48 m$ & 1 & 1 \\
\hline$\geq 49 \mathrm{~m}$ & $1,719(0,844-3,5)$ & $1,581(0,7-3,574)$ \\
\hline \multicolumn{3}{|l|}{$\mathrm{Hb}$} \\
\hline$\leq 9 \mathrm{~g} / \%$ & $1,177(0,657-2,109)$ & $0,928(0,47-1,832)$ \\
\hline$\geq 9,1$ & 1 & 1 \\
\hline \multicolumn{3}{|l|}{$\mathrm{Kt} / \mathrm{V}$} \\
\hline$\leq 0,9$ & $3,955(1,069-11,012)$ & $4,072(1,182-14,031)^{*}$ \\
\hline 0,91 a 1,2 & 1 & 1 \\
\hline$\geq 1,21$ & $1,215(0,0753-2,317)$ & $1,175(0,572-2,415)$ \\
\hline
\end{tabular}

*Estatisticamente significante ao nível de $5 \%$.

m: meses; Hb: hemoglobina; Kt/V: clearance fracional de uréia.

\section{Discussão}

As dificuldades psicossociais apresentadas por pacientes portadores de IRC têm sido alvo de interesse crescente nas últimas décadas. Isto decorre principalmente da ênfase cada vez maior dada à qualidade de vida desses indivíduos. Os aspectos psiquiátricos como contribuintes para a qualidade de vida adquirem grande importância no diagnóstico e nas 
estratégias de tratamento desses pacientes, visto que a alta prevalência de transtornos psiquiátricos está associada a pior prognóstico e a menor sobrevida dessa população (Finkelstein e Finkelstein, 2000; Wai et al., 1981).

Com este trabalho pretendeu-se preencher uma lacuna, uma vez que raros são os estudos que avaliam a saúde mental dos pacientes em programa de diálise no Brasil, principalmente com a utilização de entrevistas padronizadas. Em contraste com as usuais consultas clínicas não-sistematizadas, essas entrevistas possibilitam comparações entre diversos centros, têm a capacidade de reduzir a variabilidade no diagnóstico, estimulam os estudos colaborativos internacionais e promovem a adoção de critérios para diagnósticos psiquiátricos válidos (Andrews e Peters, 1998; Kutner et al., Valderrábano et al., 2001).

O questionário MINI utilizado neste estudo foi validado quando em comparação com o Structured Clinical Interview for DSM-III-R, Patient Version (SCID-P) e com o Composite International Diagnostic Interview (CIDI), dois dos mais utilizados instrumentos de diagnóstico da pesquisa psiquiátrica (Sheehan et al., 1998). Deve-se ressaltar que este é o primeiro estudo no qual se utiliza o MINI em pacientes com IRC, condição médica caracterizada por sintomas somáticos. Inconsistências no diagnóstico podem ocorrer quando da avaliação de sintomas físicos comuns em doenças crônicas como a IRC. A aplicação do MINI como entrevista se confirmou de fácil e rápida execução, pois os pacientes relataram boa compreensão de todos os módulos.

Embora não se possam extrapolar os dados aqui encontrados como representativos da população baiana e brasileira em tratamento dialítico, este estudo contribui para o conhecimento da magnitude do problema em nosso meio. Assim, significante porcentagem da nossa amostra exibiu sintomas psiquiátricos, confirmando que altas taxas desses transtornos ocorrem em unidades de hemodiálise. Revisões realizadas estimam que transtornos psiquiátricos nesses pacientes variem de $10 \%$ a $70 \%$, sendo as síndromes mais prevalentes a depressão e a ansiedade, com taxas que variam de $12 \%$ a $45 \%$ (Kutner et al., 1985; Kimmel et al., 1998).

A prevalência de $37,3 \%$ na amostra, quando considerados em conjunto os transtornos, é praticamente duas vezes a encontrada em populações gerais, conforme o estudo epidemiológico americano de 1988, o Epidemiologic Catchment Area (ECA), em que $15,4 \%$ de 18.571 indivíduos estudados prospectivamente preencheram critérios de transtorno mental em um mês (Robins e Regier, 1991).

Ao comparar os resultados do presente trabalho com - Estudo Multicêntrico Brasileiro de Morbidade Psiquiátrica baseado nos critérios estabelecidos pelo ECA, que apresentou prevalências entre $20 \%$ e $35 \%$ no último ano e $30 \%-50 \%$ quando se considerou a vida inteira, constatam-se prevalências diferentes nas categorias diagnósticas. Enquanto neste estudo os transtornos mais prevalentes foram os afetivos, no outro os mais freqüentes foram ansiedade e fobias (AlmeidaFilho et al., 1997).

Os transtornos mais freqüentes da amostra foram os afetivos. É especialmente preocupante a quantidade de pa- cientes com tendência suicida (40 indivíduos), já que esses, em tratamento, deparam-se freqüentemente com oportunidades de efetivar essas intenções.

Em metanálise publicada em 2000 foram incluídos 12 estudos com mais de 500 pacientes em diálise. Os autores concluíram que o risco de não-adesão ao tratamento dos pacientes deprimidos foi três vezes maior e, portanto, sugerem a importância de se reconhecer a depressão como fator de risco de morbidade e mortalidade entre esses pacientes (DiMatteo MR et al., 2000). Ressalta-se a baixa prevalência de abuso de tóxicos nessa amostra, o que contrasta com a prevalência no estudo multicêntrico brasileiro já referido, que reportou taxas de aproximadamente 15\% em homens quando se considerou a vida inteira (Almeida-Filho et al., 1997).

O sexo feminino apresentou prevalência quase duas vezes mais alta de transtornos psiquiátricos do que o masculino, dado confirmado em praticamente todos os estudos. Esse risco aumentado (mais de duas vezes) de ocorrência desses transtornos em mulheres, também encontrado no nosso estudo, pode ser um indicador de que elas têm dificuldades maiores de lidar com situações adversas (Wai et al., 1981).

O suporte familiar - representado neste estudo pelo estado civil - também confirma dados da literatura, sugerindo que intimidade e bom relacionamento entre o paciente e seu cônjuge são fatores importantes para minimizar a ocorrência de transtorno psiquiátrico (Finkelstein e Finkelstein, 2000; Wai et al., 1981). Em relação à idade, chama a atenção o pequeno número de pacientes com mais de 61 anos; portanto um perfil epidemiológico distinto do observado em unidades de outros países onde a prevalência de idosos é bem maior (U.S. Renal Data System, 2000).

De acordo com nossos resultados, o tempo em programa de diálise foi preditivo de melhor adaptação ao tratamento e mostrou consistência com dados da literatura, como o estudo de Craven et al. (1987), que demonstrou maior prevalência de depressão em 99 indivíduos nos primeiros dois anos do tratamento. Pacientes com IRC freqüentemente experimentam melhora nas condições médicas após o início da diálise e necessitam de algum tempo para se adaptar à nova situação, demonstrando acostumar-se ao tratamento após tempo médio de um ano (Craven et al., 1987).

Os pacientes mais bem dialisados, com a dose da diálise adequadamente prescrita e mensurada pelo $\mathrm{Kt} / \mathrm{V}$, apresentaram menor risco de transtornos psiquiátricos. 0 $\mathrm{Kt} / \mathrm{V}$ é apontado como importante indicador de mortalidade, com impacto na qualidade de vida, por estabelecer melhor controle metabólico da uremia, melhorar a morbidade cardiovascular, assim como os sintomas relacionados à diálise (Hakim et al., 1994).

Acreditamos que pesquisas sobre aspectos psicossociais, de qualidade de vida e prevalência de transtornos psiquiátricos possam exercer importante contribuição na tomada de decisões clínicas e de cuidados aos pacientes com IRC. Evidências sustentam a eficácia da psicoterapia e do tratamento farmacológico dos transtornos psiquiátricos nessa população (Finkelstein e Finkelstein, 2000; Kimmel et al., 1998). 
Esperamos que os dados e os resultados descritos possam ser úteis em estimular outras pesquisas nessa área, bem como sirvam de alerta para a alta prevalência desses transtornos no âmbito das unidades de diálise e que, finalmente, possam contribuir de alguma forma para melhorar a qualidade de vida dos pacientes portadores de IRC em programas de hemodiálise.

\section{Referências}

Almeida-Filho N, Mari JJ, Coutinho E, França JF, Fernandes J, Andreoli $\mathrm{SB}$, Busnello ED. Brazilian multicentric study of psychiatric morbidity: methodological features and prevalence estimates. Br J Psych, 171: 524-9, 1997.

Andrews $G$, Peters $L$. The psychometric properties of the Composite International Diagnostic Interview. Psychiatr Epidemiol, 33(2): 80-8, 1998

Craven GL, Rodin GM, Johnson $L$ et al. The diagnosis of major depression in renal dialysis patients. Psychosom Med, 49: 48292, 1987.

Dialysis Statistics. <http://www.globoldialysis.com/stats.asp>.

DiMatteo MR, Lepper HS. et al. Depression is a risk factor for noncompliance with medical treatment: meta-analysis of the effects of anxiety and depression on patients adherence. Arch Inter Med, 160(24): 2101-7, 2000.

Finkelstein FO, Finkelstein SH. Depression in chronic dialysis patients: assessment and treatment. Nephrol Dial Transplant, 15: 1911-3, 2000.

Hakim RM, Breyner J, Ismail $\mathrm{N}$ et al. Effects of dose of dialysis on morbidity and mortality. Am J Kidney Dis, 23: 661-9, 1994.

\section{Conclusão}

A prevalência de transtornos psiquiátricos foi alta, sendo os transtornos afetivos os mais freqüentes. Nas mulheres e naqueles com Kt/V baixo, o risco foi maior. Nos pacientes casados e naqueles com mais de dois anos de tratamento o risco foi menor.

Kimmel PL, Thamer M, Richard CM, Ray NF. Psychiatric illness in patients with end-stage renal disease. Am J Med, 105(3): 214-21, 1998.

Kutner NG, Fair PL, Kutner MH. Assessing depression and anxiety in chronic dialysis patients. J Psychom Res, 29(1): 23-31, 1985.

Lloyd G.G. Textbook of General Hospital Psychiatry. Churchill Livingstone, p. 167-8, Edinburgh, 1991.

Robins LN, Regier D, editors. Psychiatric Disorders in America: the Epidemiologic Catchment Area Study. New York: Free Press; 1991.

Sheehan DS et al. The Mini-International Neuropsychiatric Interview (MINI): the development and validation of a structured diagnostic psychiatric interview for DSM-IV and ICD-10. J Clin Psychiatry, 59(Suppl 20): 22-3, 1998.

U.S. Renal Data System. USRDS 2000 Anual Data Report: the National Institutes of Health, National Institute of Diabetes and Digestive and Kidney Diseases. Bethesda, 2000.

Valderrábano F, Jofre R, López-Gómez JM. Quality of life in end-stage renal disease patients. Am J Kidney Dis, 38(3): 443-64, 2001.

Wai L, Richmond J, Burton H, Linday RM. Influence of psychosocial factors on survival of hemodialysis patients. Lancet, 1155-6, 1981. 